\title{
Stent patency in patients with distal malignant biliary obstruction receiving chemo(radio)therapy
}

\section{(๑) $\odot \ominus$}

\author{
Authors \\ Sylke Haal, Jeanin E. van Hooft, Erik A. J. Rauws, Paul Fockens, Rogier P. Voermans
}

Institution

Department of Gastroenterology and Hepatology, Academic Medical Center, University of Amsterdam, Amsterdam, The Netherlands

submitted 8.3.2017

accepted after revision 4.7 .2017

Bibliography

DOI https://doi.org/10.1055/s-0043-117953 |

Endoscopy International Open 2017; 05: E1035-E1043

(C) Georg Thieme Verlag KG Stuttgart · New York

ISSN 2364-3722

Corresponding author

S. Haal, MD, Stadionplein 85-1, 1076 CK Amsterdam, The Netherlands

Fax: +31-20-6917033

s.haal@amc.uva.nl

\section{ABSTRACT}

Background and study aims Recent literature suggests that chemo(radio)therapy might reduce the patency of plastic stents in patients with malignant biliary obstruction. Whether this might also be valid for other types of stents is unknown. The aim of this study was to determine the influence of chemo(radio)therapy on the patency of fullycovered self-expandable metal stents (FCSEMSs) and plastic stents.
Patients and methods We retrospectively reviewed the electronic medical records of patients with distal malignant biliary obstruction who underwent biliary stent placement between April 2001 and July 2015. Primary outcome was duration of stent patency. Secondary outcome was stent patency at 3 and 6 months. We used Kaplan-Meier survival analyses to compare stent patency rates between patients who received chemo(radio)therapy and patients who did not.

Results A total of 291 biliary stents (151 metal and 140 plastic) were identified. The median cumulative stent patency of FCSEMSs did not differ between patients receiving chemo(radio)therapy $(n=51)$ and those $(n=100)$ who did not $(P=0.70$, log-rank test). The estimated cumulative stent patency of plastic stents was also comparable in 99 patients without and 41 patients with chemo(radio)therapy $(P=0.73$, log-rank test). At 3 and 6 months, FCSEMS patency rates were $87 \%$ and $83 \%$ in patients without chemo (radio)therapy and $96 \%$ and $83 \%$ in patients with therapy, respectively. Plastic patency rates were $69 \%$ and $55 \%$ in patients without and $85 \%$ and $39 \%$ in patients with therapy, respectively. After 1 year, $78 \%$ of the FCSEMSs were still patent in patients without chemo(radio)therapy and $69 \%$ of the FCSEMSs were still patent in patients with therapy.

Conclusion Our data indicate that chemo(radio)therapy does not reduce the patency of biliary fully-covered metal and plastic stents.

\section{Introduction}

In Europe and the United States, pancreatic cancer is, respectively, the 6 th and 4 th leading cause of cancer-related deaths $[1,2]$. Only $15-20 \%$ of patients are candidates for surgery, because in the majority, the cancer has already spread to other parts of the body or there is locally advanced disease at the time of diagnosis [3]. Even after complete surgical resection, long-term survival is rare as a result of local recurrence or distant metastases [4].

Therefore, chemo(radio)therapy is increasingly used in the hope of improving long-term survival. Unfortunately, the use of adjuvant chemo(radio)therapy has shown no considerable improvements in overall survival [5]. Neoadjuvant therapy seems to offer the possibility of downstaging borderline resectable tumors into resectable tumors [6]. And secondly, it implies enhanced survival in the case of resectable tumors by increasing the $\mathrm{R} 0$ resection rate [7].

Approximately $70 \%$ of patients with periampullary tumors develop jaundice during the course of their disease due to malignant biliary obstruction [8]. Chemotherapy requires a normal liver function to avoid toxicity and therefore, adequate biliary drainage [9]. Endoscopic biliary retrograde cholangiopancreaticography (ERCP) with biliary stent placement is the preferred 
method over percutaneous stent placement and surgical bypass $[10,11]$. Accumulating evidence shows that self-expandable metal stents (SEMSs) are superior to plastic stents in terms of stent patency [12].

In theory, chemo(radio)therapy might reduce stent patency, as immunosuppression can result in bacterial colonization of the stent [13-16]. Plastic stents in particular might be susceptible to early stent dysfunction during chemo(radio)therapy, as biofilm and sludge formation are the main causes of plastic stent dysfunction, while fully-covered self-expandable metal stents (FCSEMSs) are prone to migration [17-19]. Several clinical studies have supported this hypothesis by showing that the use of chemo(radio)therapy reduces the patency of plastic stents, while SEMSs seem to maintain patency during the time required for patients to complete neoadjuvant therapy [1315, 20 -24]. However, other studies could not establish an effect of chemo(radio)therapy on either plastic or metal stent patency $[16,25,26]$. Because of these heterogeneous results, we sought to clarify the influence of chemo(radio)therapy on biliary stents in this retrospective study. Therefore, we investigated the stent patency rates of FCEMSs and plastic stents in patients with distal malignant biliary obstruction who received chemo(radio)therapy and patients who did not receive chemo (radio)therapy. We hypothesized that the patency of both stent types is not reduced by chemo(radio)therapy.

\section{Patients and methods}

\section{Study design and patients}

We performed a retrospective analysis of a prospective endoscopic database (Endobase: Olympus Medical Systems Europe, Hamburg, Germany) in the Academic Medical Center (AMC), a tertiary referral center in the Netherlands. The Medical Ethics Review Committee of the Academic Medical Center in Amsterdam confirmed that the Dutch Medical Research Involving Human Subjects Act did not apply to the current study and that an official approval was not required.

We identified patients who had undergone biliary drainage with a FCSEMS or plastic stent for malignant biliary obstruction. We included patients who were treated with chemo(radio)therapy between April 2001 and July 2015. Patients who had undergone plastic stent placement and who did not receive chemo (radio)therapy were included between July 2010 and July 2015. Reports of the endoscopic procedures were reviewed to identify patients with distal common bile duct obstruction caused by pancreatic cancer or distal cholangiocarcinoma. All patients were included regardless of tumor resectability. Furthermore, we included patients who received neoadjuvant therapy as well as palliative therapy. The oncologic therapy regimes were chosen by an oncologist and the majority of patients participated in clinical trials. Patients with malignant obstruction at the level of the hilum caused by a proximal cholangiocarcinoma or gallbladder malignancy, were excluded from the study because hilar obstructions cannot be drained with FCSEMSs. Ampullary carcinomas were excluded because chemo(radio)therapy is not recommended for this type of tumor. Moreover, patients were excluded if they received an uncov- ered SEMS. Finally, we excluded patients who had undergone percutaneous biliary drainage simultaneously.

\section{Data collection}

In order to collect data, we reviewed stent placement procedures and electronic medical records for all selected patients. We collected data on patient demographics (gender, age) and tumor characteristics which included type of tumor, resectability and presence of histological or cytological confirmation of the diagnosis. All endoscopic procedures were performed or supervised by an experienced therapeutic endoscopist. Stent type, length and diameter were at the discretion of the endoscopist. Furthermore, we collected data on the type and timing of chemo(radio)therapy, duration of stent patency, stent patency at 3 and 6 months after placement, and the reason for stent exchange. In order to collect all data, we contacted referral centers if a patient received local care after stent insertion. Likewise, we contacted general practitioners to determine the day of death when electronic medical records lacked this information in our institution.

\section{Study outcomes}

The primary outcome was the duration of stent patency. Stent patency was defined as the time between stent placement and stent exchange due to dysfunction. Stent dysfunction was suspected when patients presented with increased cholestasis or cholangitis necessitating stent exchange by ERCP as judged by the clinician. Secondary outcome was plastic stent and FCSEMS patency at 3 and 6 months and FCSEMS patency at 1 year.

\section{Follow-up}

Patients were followed until they underwent elective stent exchange, experienced stent dysfunction, died, or underwent surgical pancreatoduodenectomy or palliative hepaticojejunostomy. If none of these end points had occurred, the followup ended 1 year after stent insertion.

\section{Statistical analysis}

Each stent placement was recorded as an independent event, considering that each patient may have received multiple stents during the course of their treatment. Descriptive statistics were used to describe the population. Continuous variables with a normal distribution were summarized using means and standard deviations (SD), whereas medians and interquartile ranges (IQR) or ranges were used for skewed distributions. Categorical variables were summarized using numbers and percentages. Comparisons between the groups with and without chemo(radio)therapy were performed with the independent samples $t$ test for continuous variables with a normal distribution. The Chi-squared test was used for categorical variables and Fisher's exact test in cases where cell count was less than five. A probability $(P)$ value of 0.05 or smaller was considered to imply statistical significance and all reported $P$ values are two-sided. Cumulative stent patency rates were estimated using Kaplan-Meier analysis and life table analyses. Censored cases included patients who underwent elective stent exchange, patients who underwent stent removal during surgery, 
and patients who had died before stent dysfunction had occurred within 1 year of follow-up. In addition, patients were censored in cases when stent dysfunction did not occur within 1 year after stent insertion. The log-rank test was used to compare the cumulative stent patency of FCSEMSs and plastic stents with and without chemo(radio)therapy. All analyses were performed using SPSS software version 22 (SPSS, Inc, Chicago, Illinois, United States).

\section{Results}

\section{Baseline characteristics}

In total, 266 patients were included in this study, and a total of 291 biliary stents were placed between April 2001 and July 2015. Of these, 151 were FCSEMSs and 140 were plastic stents (10 French). Baseline characteristic are summarized in $>$ Table 1. The mean age of the overall cohort was $65.6 \pm 10.3$ years, and $185(63.6 \%)$ stents were placed in male patients. The majority of patients $(82.8 \%)$ suffered from pancreatic cancer, and a tissue diagnosis was present in $88.3 \%$ of all patients. About onethird $(35.1 \%)$ of the stents were placed in patients with resectable tumors and 92 of 291 stents (31.6\%) were placed in patients receiving chemo(radio)therapy. The majority (71.1\%) were treated with gemcitabine-based chemo(radio)therapy (see $>$ Supplementary Table 1 for details with regard to type of chemotherapy).

Apart from tumor resectability, there were no differences in baseline characteristics between the two groups with FCSEMSs. In the case of plastic stents, patients with chemo(radio)therapy were younger, suffered more frequently from pancreatic cancer, and the tumors were more often unresectable ( $\triangleright$ Table 1 ).

\section{Stent patency}

The primary outcome per group is summarized in $>$ Table 2 . The overall median patency of FCSEMSs without chemotherapy was 36 days [IQR 21 - 90 days]. In total, 44 patients (44\%) underwent surgery after a median preoperative drainage time of 29 days [IQR $22-38$ days]. Ten stents (10\%) were exchanged prematurely after a median of 16 days [IQR 8 - 91 days]. Premature exchange was performed in the case of stent dysfunction. Reasons for stent dysfunction are included in $>$ Table 3 . The overall median patency of FCSEMSs in patients receiving chemo(radio)therapy was 191 days [IQR 119-365 days]. Eight stents $(15.7 \%)$ were removed at surgery after 123 days [IQR $107-157$ ] and 10 stents (19.6\%) were exchanged prematurely after a median of 126 days [IQR 96-291 days]. A total of 12 FCSEMSs were still patent after maximum follow-up of 1 year in the group of patients who were treated with chemo(radio) therapy.

The overall median patency of plastic stents was 38 days [IQR 23-58 days] in patients who did not receive chemo (radio)therapy. Twenty-four stents (24.2\%) were exchanged prematurely after a median of 38 days [IQR 19-88 days]. Finally, in the group of plastic stents with chemo(radio)therapy, the overall median patency was 133 days [IQR $96-214$ days]. In this group, 27 stents (65.9\%) were exchanged prematurely after a median of 127 days [IQR $91-157$ days].

\section{Cumulative stent patency}

The Kaplan-Meier curves for estimated cumulative stent patency are shown in $>$ Fig. 1. Ninety percent of the FCSEMSs without chemo(radio)therapy and $80.4 \%$ of the FCSEMSs with chemo(radio)therapy were censored. A more detailed overview of the censored cases is presented in $\mathbf{F i g . 2}$. The estimated cumulative stent patency of FCSEMSs did not differ between patients receiving chemo(radio)therapy and those who did not ( $P=0.70$, log-rank test). Both medians were not reached. The rate of censored cases in the group of plastic stents without chemo(radio)therapy was $75.8 \%$, and $34.1 \%$ in the group with chemo(radio)therapy ( $>$ Fig. 2 ). The estimated cumulative stent patency of plastic stents was comparable in patients with (median: 144 days [95\%Cl:125-163]) and without (median: 182 days [95\% Cl: $103-261])$ chemo(radio)therapy $(P=0.73$, log-rank test).

\section{Cumulative stent patency at 3 and 6 months}

Life table analysis showed FCSEMS patency rates of $87 \%$ at 3 months, $83 \%$ at 6 months and $78 \%$ at 1 year in patients who did not receive chemo(radio)therapy. In patients who were treated with chemo(radio)therapy, the patency rates of FCSEMSs were $96 \%$ at 3 months, $83 \%$ at 6 months, and $69 \%$ at 1 year. Plastic patency rates were $69 \%$ and $55 \%$ at, respectively 3 and 6 months in patients without chemo(radio)therapy, and $85 \%$ and $39 \%$ in patients with chemo(radio)therapy.

\section{Discussion}

This retrospective study determined the potential influence of chemo(radio)therapy on the patency of biliary stents in patients with malignant biliary obstruction. We hypothesized that the patency of FCSEMSs and plastic stents is not reduced by chemo(radio)therapy. Our data confirmed this hypothesis by revealing no difference in cumulative stent patency rates for FCSEMSs, as well as plastic stents.

After 3 months, $96 \%$ of the FCSEMSs were still patent in patients with chemo(radio)therapy, and $87 \%$ of the FCSEMSs in patients without therapy ( $\triangleright$ Fig. 1). After 6 months, stent patency rates were $83 \%$ in both groups. These stent patency rates are clinically relevant since most neoadjuvant therapy regimes last approximately 3-6 months. In an earlier retrospective analysis of 80 patients who underwent palliative chemo(radio) therapy, life table analysis showed similar FCSEMS patency rates of $97 \%$ at 3 months and $85 \%$ at 6 months [27]. Our study further expanded the literature, because we also provided data showing that patency rates of FCSEMSs in patients who did not receive chemo(radio)therapy during the same time frame, did not differ. Furthermore, two earlier Japanese studies support our data, because they found no effect of chemotherapy on metal stent patency $[25,26]$.

To the best of our knowledge, only one small prospective randomized study has been conducted with regard to FCSEMS patency in patients with a malignant obstruction, receiving neoadjuvant therapy. In that study, 54 patients were randomized to receive a FCSEMS, uncovered SEMS (USEMS) or plastic 


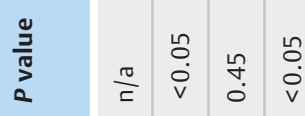

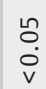

$\frac{\pi}{\check{2}}$

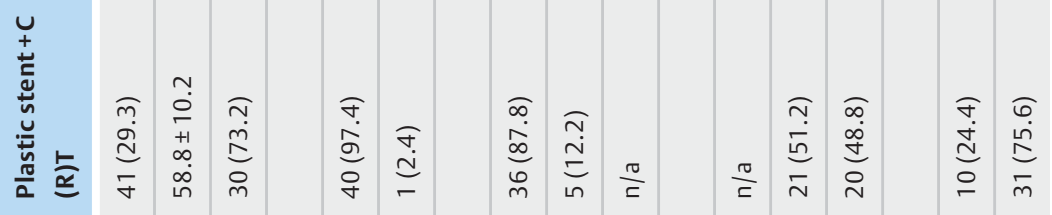

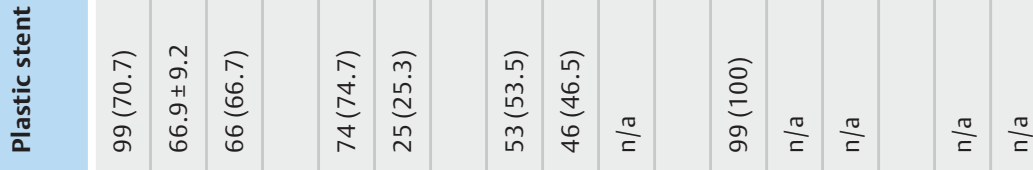

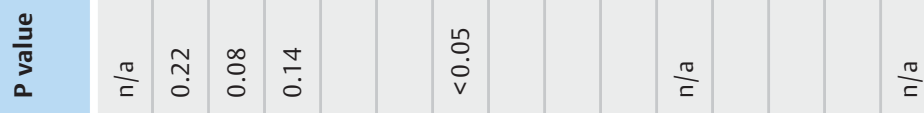

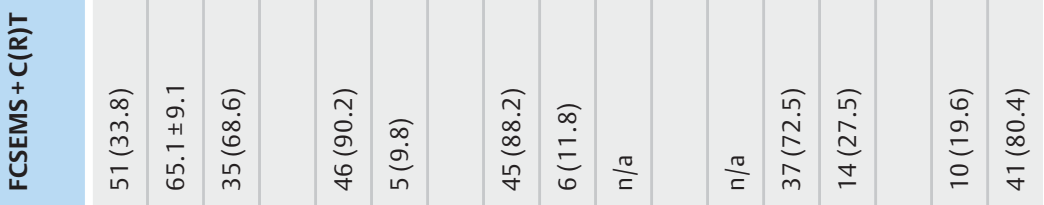

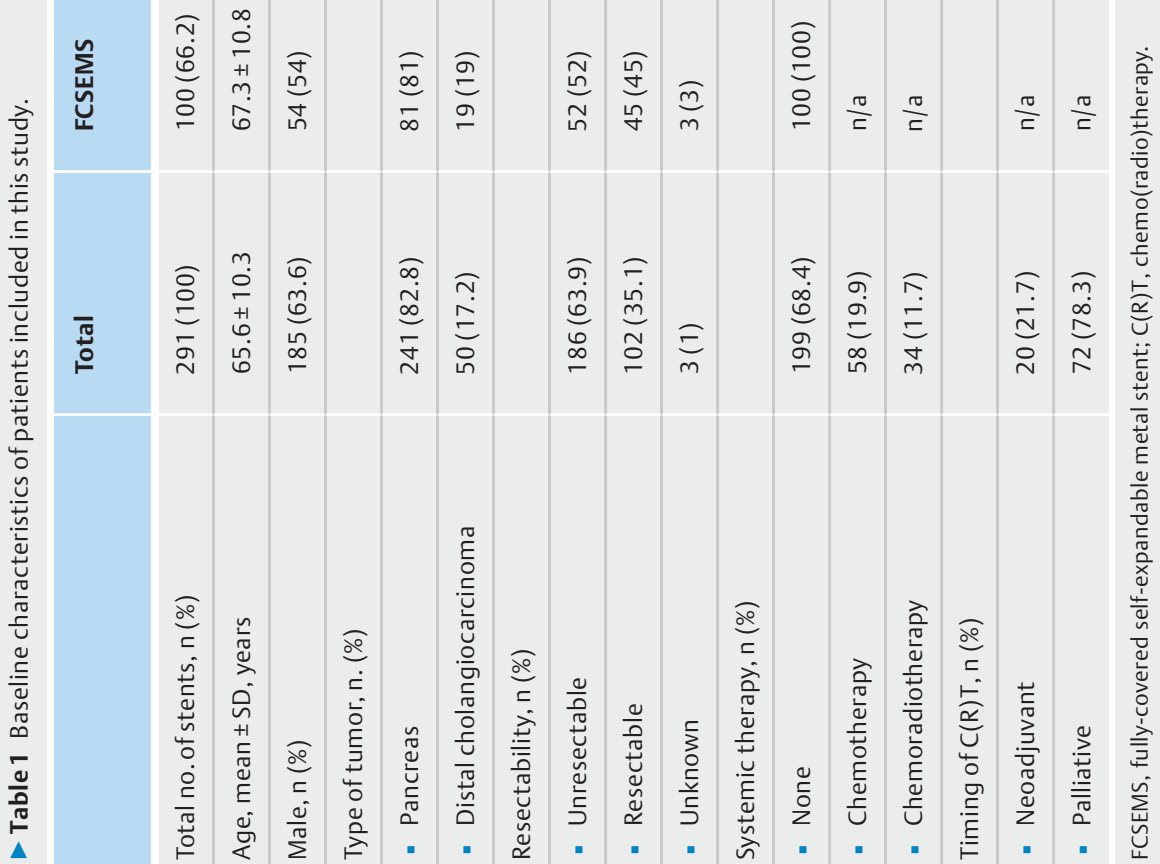


- Table 2 Stent patency data.

\begin{tabular}{|c|c|c|}
\hline & $\begin{array}{l}\text { Stents placed, } \mathbf{n} \\
\text { (\%) }\end{array}$ & $\begin{array}{l}\text { Median patency, } \\
\text { days, [IQR] } \\
\text { or (range) }{ }^{1}\end{array}$ \\
\hline \multicolumn{3}{|l|}{ FCSEMS } \\
\hline Total no. of stents & $100(100)$ & $36[21-90]$ \\
\hline Elective exchange & $1(1)$ & 73 \\
\hline Premature exchange & $10(10)$ & $16[8-91]$ \\
\hline \multicolumn{3}{|l|}{ Others } \\
\hline - Removed at surgery & $44(44)$ & $29[22-38]$ \\
\hline - Death & $38(38)$ & $80[20-177]$ \\
\hline - End of follow-up² & $7(7)$ & 365 \\
\hline \multicolumn{3}{|l|}{ FCSEMS + C(R)T } \\
\hline Total no. of stents & $51(100)$ & $191[119-365]$ \\
\hline Elective exchange & $\mathrm{n} / \mathrm{a}$ & $\mathrm{n} / \mathrm{a}$ \\
\hline Premature exchange & $10(19.6)$ & 126 [96-291] \\
\hline \multicolumn{3}{|l|}{ Others } \\
\hline - Removed at surgery & $8(15.7)$ & $123[107-157]$ \\
\hline - Death & $21(41.2)$ & 191 [112-249] \\
\hline - End of follow-up & $12(23.5)$ & 365 \\
\hline \multicolumn{3}{|l|}{ Plastic stent } \\
\hline Total no. of stents & $99(100)$ & $38[23-58]$ \\
\hline Elective exchange & $2(2)$ & $48(41-54)$ \\
\hline Premature exchange & $24(24.2)$ & $38[19-88]$ \\
\hline \multicolumn{3}{|l|}{ Others } \\
\hline - Removed at surgery & $55(55.6)$ & $34[22-46]$ \\
\hline - Death & $17(17.2)$ & $112[51-195]$ \\
\hline - End of follow-up ${ }^{2}$ & $1(1)$ & 365 \\
\hline \multicolumn{3}{|l|}{ Plastic stent $+C(R) T$} \\
\hline Total no. of stents & $41(100)$ & $133[96-214]$ \\
\hline Elective exchange & $2(4.9)$ & $201(126-276)$ \\
\hline Premature exchange & 27 (65.9) & 127 [91-157] \\
\hline \multicolumn{3}{|l|}{ Others } \\
\hline - Removed at surgery & $3(7.3)$ & $94[41-133]$ \\
\hline - Death & $8(19.5)$ & $183[135-263]$ \\
\hline - End of follow-up² & $1(2.4)$ & 365 \\
\hline \multicolumn{3}{|c|}{$\begin{array}{l}\text { FCSEMS, fully-covered self-expandable metal stent; } C(R) T \text {, chemo(radio) } \\
\text { therapy; IQR, interquartile range. } \\
{ }^{1} \text { Range: is used in the case of insufficient data for constructing IQR. } \\
{ }^{2} \text { One year after stent insertion. }\end{array}$} \\
\hline
\end{tabular}

therapy; IQR, interquartile range.

${ }^{1}$ Range: is used in the case of insu
${ }^{2}$ One year after stent insertion. stent. The outcome variables of interest were the time to stent occlusion, attempted surgical resection or death after the initiation of neoadjuvant therapy. The mean time to stent occlusion was significantly longer in FCSEMSs compared with USEMSs and plastic stents ( 220 days vs 74 days and 76 days, $P$ $<0.01$ ) [24]. However, the use of mean times to stent occlusion represents a statistical limitation. Stent occlusion was only observed in 21 patients because others underwent surgery ( $\mathrm{n}=$ 18 ) or had died $(n=15)$ before stent occlusion had occurred. Calculating the mean time to stent occlusion with only the measurements of patients who experienced stent occlusion could have underestimated or overestimated the time to stent occlusion. On the other hand, Kaplan-Meier survival analysis enables all of the information available to be used by taking account of the patients who underwent surgery or died when estimating the cumulative stent patency time. Furthermore, that study lacked controls who did not receive chemo(radio)therapy.

Additionally, our data did not show a difference in cumulative patency of plastic stents between patients who did and who did not receive chemo(radio)therapy. However, the premature stent exchange rates, $24.2 \%$ in patients without chemo(radio)therapy vs. $65.9 \%$ in patients with chemo(radio)therapy, respectively, seem to be inconsistent with the previously drawn conclusion ( $\$$ Table 2 ). But the discrepancy between the low premature exchange rate in patients without therapy and the high rate in patients with therapy can be explained by the fact that more than half of the stents in the group without chemo(radio)therapy were removed during surgery within 1 month. Hence, these stents never had the chance to become occluded.

At least two earlier studies refuted the claim that chemo (radio)therapy might reduce the patency of plastic stents $[16,28]$. Our results confirm the findings of an earlier small trial by Lofts et al. [16]. They performed a retrospective study in 80 patients with a malignant biliary obstruction caused by heterogeneous primary tumors of whom 39 received a plastic biliary stent. The median stent patency in that study did not differ between patients with and without chemotherapy (105 vs. 119 days, respectively). On the other hand, a recently published trial suggested that chemo(radio)therapy reduces the patency of plastic biliary stents [13]. That retrospective trial included 173 patients with borderline resectable pancreatic cancer who underwent neoadjuvant chemo(radio)therapy. A total of 233 plastic stents were placed, and $35.6 \%$ of the plastic stents were exchanged prematurely after a median of 49 days. The authors suggested shortening the interval for elective exchange, as only $34.3 \%$ of the plastic stents remained patent at the recommended exchange interval of 3 months. Unfortunately, that study lacked a control group of patients who did not receive chemo(radio)therapy during the same time frame. Another retrospective study $(n=49)$ concluded similarly to Ge et al. that plastic stents do not maintain patency during the time required for completing neoadjuvant therapy. However, stent patency rates at 3 and 6 months were not provided in that study [20]. 
- Table 3 Reasons for stent dysfunction.

\begin{tabular}{|c|c|c|c|c|}
\hline & $\begin{array}{l}\text { FCSEMS } \\
(n=10)\end{array}$ & $\begin{array}{l}\text { FCSEMS + C(R)T } \\
(n=10)\end{array}$ & $\begin{array}{l}\text { Plastic stent } \\
(n=24)\end{array}$ & $\begin{array}{l}\text { Plastic stent }+C(R) T \\
(n=27)\end{array}$ \\
\hline Sludge formation & 4 & 2 & 11 & 12 \\
\hline Stent migration & 4 & 6 & 5 & 3 \\
\hline Tumor overgrowth & 1 & 2 & 0 & 0 \\
\hline Other $^{1}$ & 1 & 0 & 2 & 0 \\
\hline Unknown & 0 & 0 & 6 & 12 \\
\hline
\end{tabular}

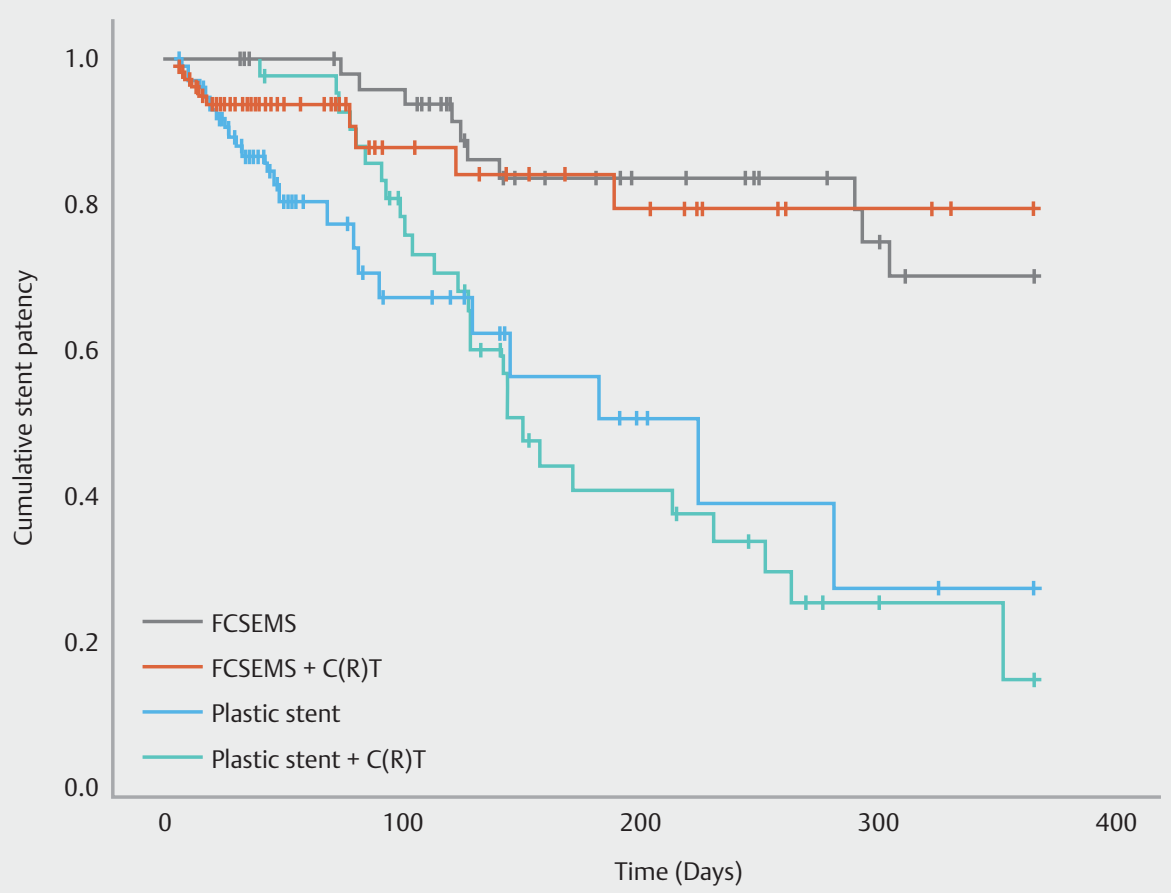

No. of remaining cases

\begin{tabular}{lccccccccc}
\hline FCSEMS & 100 & 40 & 24 & 19 & 16 & 11 & 9 & 7 & \\
FCSEMS + C(R)T & 51 & 48 & 45 & 29 & 23 & 19 & 16 & 13 \\
\hline Plastic & 99 & 33 & 17 & 9 & 6 & 3 & 2 & 1 \\
Plastic stent + C(R)T & 41 & 39 & 29 & 15 & 11 & 7 & 3 & 2
\end{tabular}

- Fig. 1 Kaplan-Meier curves showing cumulative stent patency. FCSEMS: fully-covered self-expandable metal stent; C(R)T: chemo(radio)therapy.

The median stent patency rates shown in $>$ Table 2 require some clarification. We considered the median stent patency less appropriate to compare stent patency rates between groups with and without chemo(radio)therapy than estimations of the cumulative stent patency ( $\mathbf{F i g . 1}$ ). However, the median stent patency rates are shown because they provide extra insights, even though the outcomes are biased due to an unequal patient distribution. The short overall median stent patency durations in patients without therapy (36 days with
FCSEMSs and 38 days with plastic stents) most likely reflect the shorter preoperative drainage times in these patient groups as patients without chemo(radio)therapy underwent immediate surgery, whereas patients with chemo(radio)therapy underwent surgery after completion of neoadjuvant therapy. Also the surprisingly short patency of prematurely exchanged stents in patients without chemo(radio)therapy needs explanation: 16 days with FCSEMSs and 38 days with plastic stents. Of all placed stents $(n=291), 14$ were prematurely exchanged within 3 

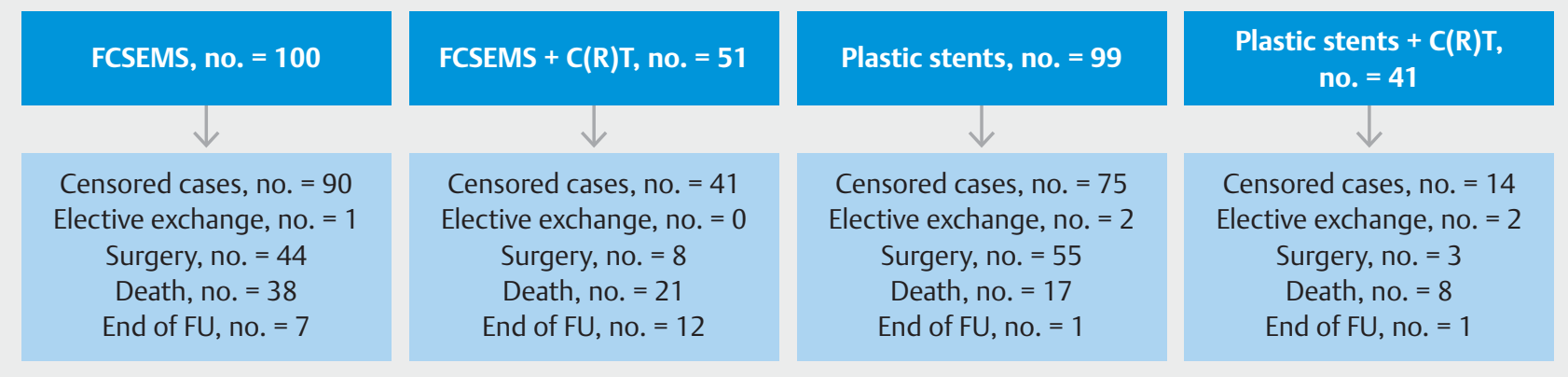

- Fig. 2 Overview of censored cases. FCSEMS: fully-covered self-expandable metal stent; C(R)T: chemo(radio)therapy; FU: follow-up.

weeks after placement, mainly due to persistent cholestasis caused by early stent migration or persistent biliary sludge. Even if they would have been scheduled for chemotherapy, the patients who received these stents were likely to end up in the non-chemo(radio)therapy groups, since inadequate biliary drainage prohibited these patients from starting chemotherapy.

The abovementioned results should be interpreted taking into account several limitations. First, the retrospective nature of data collection is subject to bias, and its nonrandomized nature caused inequalities in patient characteristics. The second limitation of this study concerns the statistical analysis. We had to take the presence of many censored cases into account and the retrospective data collection contributed to a different distribution of censored cases across the groups. A final possible limitation is that we might have missed events of stent dysfunction or elective stent exchange if a patient received local care after stent insertion. Consequently, this could have caused overestimation of stent patency.

\section{Conclusion}

Our retrospective data indicate that chemo(radio)therapy does not reduce the patency of biliary stents. Furthermore, our data do not support the suggestion to shorten the regular intervals for stent exchange in patients with malignant biliary obstruction receiving chemo(radio)therapy.

\section{Competing interests}

None

\section{References}

[1] Ferlay J, Steliarova-Foucher E, Lortet-Tieulent J et al. Cancer incidence and mortality patterns in Europe: Estimates for 40 countries in 2012. Eur J Cancer 2013; 49: 1374 - 1403

[2] Siegel RL, Miller KD, Jemal A. Cancer statistics, 2015. CA Cancer J Clin 2015; 65: 5-29

[3] SEER Cancer Statistics Factsheets: Pancreas Cancer. Bethesda, Maryland: National Cancer Institute; 2015: Available from: http://seer. cancer.gov/statfacts/html/pancreas.html Accessed: 25 October 2015

[4] Sperti C, Pasquali C, Piccoli A et al. Recurrence after resection for ductal adenocarcinoma of the pancreas. World J Surg 1997; 21: 195200

[5] Oettle H, Post S, Neuhaus P et al. Adjuvant chemotherapy with gemcitabine vs observation in patients undergoing curative-intent resection of pancreatic cancer. JAMA 2007; 297: 267-277

[6] Gillen S, Schuster T, Meyer ZumBüschenfelde $C$ et al. Preoperative/ neoadjuvant therapy in pancreatic cancer: a systematic review and meta-analysis of response and resection percentages. PLoS Med 2010; 7: e1000267

[7] Artinyan A, Anaya DA, McKenzie S et al. Neoadjuvant therapy is associated with improved survival in resectable pancreatic adenocarcinoma. Cancer 2011; 117: 2044-2049

[8] Lillemoe KD, Pitt HA. Palliation. Surgical and otherwise. Cancer 1996; 78: $605-614$

[9] Eklund JW, Trifilio S, Mulcahy MF. Chemotherapy dosing in the setting of liver dysfunction. Oncology (Williston Park) 2005; 19: 1057 - 1063 ; discussion 1063-1064, 1069

[10] Speer AG, Cotton PB, Russel RC et al. Randomised trial of endoscopic versus percutaneous stent insertion in malignant obstructive jaundice. Lancet 1987; 2: 57-62

[11] Smith AC, Dowsett JF, Russell RC et al. Randomised trial of endoscopic stenting versus surgical bypass in malignant low bileduct obstruction. Lancet 1994; 344: 1655- 1660

[12] Almadi MA, Barkun A, Martel M. Plastic vs. self-expandable metal stents for palliation in malignant biliary obstruction: a series of metaanalyses. Am J Gastroenterol 2017; 112: 260 - 273

[13] Ge PS, Hamerski CM, Watson RR et al. Plastic biliary stent patency in patients with locally advanced pancreatic adenocarcinoma receiving downstaging chemotherapy. Gastrointest Endosc 2015; 81: 360 - 366

[14] Adams MA, Anderson MA, Myles JD et al. Self-expanding metal stents (SEMS) provide superior outcomes compared to plastic stents for pancreatic cancer patients undergoing neoadjuvant therapy. J Gastrointest Oncol 2012; 3: 309-313

[15] Aadam AA, Evans DB, Khan A et al. Efficacy and safety of self-expandable metal stents for biliary decompression in patients receiving 
neoadjuvant therapy for pancreatic cancer: a prospective study. Gastrointest Endosc 2012; 76: 67-75

[16] Lofts FJ, Evans TR, Mansi JL et al. Bile duct stents: is there an increased rate of complications in patients receiving chemotherapy? Eur J Cancer 1997; 33: 209-213

[17] Dumonceau JM, Tringali A, Blero D et al. Biliary stenting: Indications, choice of stents and results: European Society of Gastrointestinal Endoscopy (ESGE) clinical guideline. Endoscopy 2012; 44: 277-298

[18] Boulay BR, Parepally M. Managing malignant biliary obstruction in pancreas cancer: Choosing the appropriate strategy. World J Gastroenterol 2014; 20: 9345 - 9353

[19] Walter D, van Boeckel PG, Groenen MJ et al. Cost efficacy of metal stents for palliation of extrahepatic bile duct obstruction in a randomized controlled trial. Gastroenterology 2015; 149: 130-138

[20] Boulay BR, Gardner TB, Gordon SR. Occlusion rate and complications of plastic biliary stent placement in patients undergoing neoadjuvant chemoradiotherapy for pancreatic cancer with malignant biliary obstruction. J Clin Gastroenterol 2010; 44: 452-455

[21] Wasan SM, Ross WA, Staerkel GA et al. Use of expandable metallic biliary stents in resectable pancreatic cancer. Am J Gastroenterol 2005; 100: $2056-2061$

[22] Siddiqui AA, Mehendiratta V, Loren D et al. Self-expanding metal stents (SEMS) for preoperative biliary decompression in patients with resectable and borderline-resectable pancreatic cancer: outcomes in 241 patients. Dig Dis Sci 2013; 58: 1744-1750
[23] Kubota K, Sato T, Watanabe S et al. Covered self-expandable metal stent deployment promises safe neoadjuvant chemoradiation therapy in patients with borderline resectable pancreatic head cancer. Dig Endosc 2014; 26: $77-86$

[24] Gardner TB, Spangler CC, Byanova KL et al. Cost-effectiveness and clinical efficacy of biliary stents in patients undergoing neoadjuvant therapy for pancreatic adenocarcinoma in a randomized controlled trial. Gastrointest Endosc 2016; 84: 460-466

[25] Nakai Y, Isayama H, Kawabe T et al. Efficacy and safety of metallic stents in patients with unresectable pancreatic cancer receiving gemcitabine. Pancreas 2008; 37: 405-410

[26] Kitano M, Yamashita Y, Tanaka K et al. Covered self-expandable metal stents with an anti-migration system improve patency duration without increased complications compared with uncovered stents for distal biliary obstruction caused by pancreatic carcinoma: a randomized multicenter trial. Am J Gastroenterol 2013; 108: 1713-1722

[27] Kahaleh M, Brock A, Conaway MR et al. Covered self-expandable metal stents in pancreatic malignancy regardless of resectability: a new concept validated by a decision analysis. Endoscopy 2007; 39 : $319-324$

[28] Takasawa O, Fujita N, Kobayashi G et al. Endoscopic biliary drainage for patients with unresectable pancreatic cancer with obstructive jaundice who are to undergo gemcitabine chemotherapy. World J Gastroenterol 2006; 12: 7299-7303 
- Supplementary Table 1 Overview of chemotherapy regimes.

\begin{tabular}{|c|c|c|}
\hline & $\begin{array}{l}\text { FCSEMS + C(R)T } \\
\text { n (\%) }\end{array}$ & $\begin{array}{l}\text { Plastic stent }+C(R) T \\
n(\%)\end{array}$ \\
\hline Gemcitabine & $8(15.7)$ & $10(24.4)$ \\
\hline Gemcitabine + cisplatin & $3(5.9)$ & $\mathrm{n} / \mathrm{a}$ \\
\hline Gemcitabine + capecitabine & $\mathrm{n} / \mathrm{a}$ & $1(2.4)$ \\
\hline Gemcitabine + erlotinib & $\mathrm{n} / \mathrm{a}$ & $1(2.4)$ \\
\hline Gemcitabine + erlotinib + metformin/placebo & $9(17.6)$ & $1(2.4)$ \\
\hline Gemcitabine + erlotinib + bevacizumab/placebo & $\mathrm{n} / \mathrm{a}$ & $1(2.4)$ \\
\hline Gemcitabine + nadroparin & $3(5.9)$ & $2(4.9)$ \\
\hline Gemcitabine + etalocib/placebo & $\mathrm{n} / \mathrm{a}$ & $2(4.9)$ \\
\hline Gemcitabine + RT & $9(17.6)$ & $7(17.1)$ \\
\hline Gemcitabine + nelfinavir + RT & $1(2)$ & $5(12.2)$ \\
\hline Gemcitabine + panitumumab + RT & $1(2)$ & $\mathrm{n} / \mathrm{a}$ \\
\hline Folfirinox & $11(21.6)$ & $1(2.4)$ \\
\hline Capecitabine + everolimus & $\mathrm{n} / \mathrm{a}$ & $1(2.4)$ \\
\hline Capecitabine + celecoxib + nadroparin & $1(2)$ & $\mathrm{n} / \mathrm{a}$ \\
\hline $5 F U$ & $\mathrm{n} / \mathrm{a}$ & $1(2.4)$ \\
\hline $5 F U+R T$ & $\mathrm{n} / \mathrm{a}$ & $1(2.4)$ \\
\hline $5 \mathrm{FU}+$ leucovorin + RT & $\mathrm{n} / \mathrm{a}$ & $1(2.4)$ \\
\hline $5 \mathrm{FU}+$ leucovorin + celecoxib + RT & $3(5.9)$ & $6(14.6)$ \\
\hline Unknown & $2(3.9)$ & $\mathrm{n} / \mathrm{a}$ \\
\hline Total & $51(100)$ & $41(100)$ \\
\hline
\end{tabular}

